\title{
Malik`s Technique of Single Loop Fixation of Posterior Chamber Intraocular Lens in Presence of Partial Capsular Support
}

\author{
Krishan Pal Singh Malik ${ }^{1}$, Ruchi Goel ${ }^{*}, 2$, Divya Kishore ${ }^{2}$ and Smriti Nagpal ${ }^{2}$ \\ ${ }^{1}$ Subharti Medical College Meerut, Uttar Pradesh, India \\ ${ }^{2}$ Maulana Azad Medical College, New Delhi, India
}

\begin{abstract}
Single loop fixation of posterior chamber intraocular lens in the presence of partial capsular support is usually performed by creation of additional scleral flap or tunnel. This extra port may expose the suture holding the intraocular lens or the tucked-in lens haptics to the outside environment thereby increasing the risk of endophthalmitis. We describe a technique of single loop fixation where the scleral tunnel is created adjacent to the site with the absent capsule, the leading haptic is placed on the capsular scaffold, the trailing haptic is tied to 9-0 polypropylene, and the suture is then secured to the inner edge of the scleral lip with enough tension to center the optics and the wound is then closed. The suture knot gets buried within the scleral tunnel with no external communication and does not require a separate port. It is an easy, safe, fast and reproducible technique with a lens tilt of less than $2^{\circ}$.
\end{abstract}

Keywords: One haptic, scleral fixation, single loop, suture fixation.

\section{INTRODUCTION}

Scleral fixation of posterior chamber intraocular lens (PCIOL) is routinely practiced in the absence of sufficient capsular support. The two haptics are fixed under partially dissected scleral flaps or scleral tunnel. But this additional scleral dissection serves as a potential source of communication between the intra and extra-ocular environments and predisposes to endophthalmitis, increases the duration of surgery, and makes it cumbersome [1].

In the presence of capsular remnants, a single loop fixation has recently been reported with good outcomes [2, 3]. However, in both of these studies the haptic was fixed after creating an extra scleral wound.

Here we describe a method of a single loop fixation through the main wound itself where the haptic is fixed to the inner lip of the sclera using 9-0 polypropylene suture.

\section{PROCEDURE}

Aphakic eyes with at least $50 \%$ of intact peripheral capsular support are chosen for this procedure. Under a local or general anaesthesia in children, conjunctival peritomy is performed adjacent to the area of the absent capsule. A sclerocorneal tunnel is dissected followed by creation of a side port and an injection of viscoelastic, sodium hyaluronate $(10 \mathrm{mg} / \mathrm{ml})$. A cleavage is created between the iris and the capsular remnants by injecting viscoelastic. The anterior chamber is entered and automated vitrectomy is performed, if required. A 9-0 polypropylene monofilament suture (Aurolab AS-160-06, diameter .15mm) with a curved

*Address correspondence to this author at the EC 400, Maya Enclave, Hari Nagar, New Delhi 110064, India; Tel: 9811305645 ;

E-mail: gruchi1@rediffmail.com needle is tied to the eyelet of the trailing haptic of the scleral fixated lens (Aurolens 3C6530, 6.5mm, 'modified C' loop). A knuckle of the iris is pulled out of the wound. The tip of the curved needle with a 9-0 polypropylene suture is fastened to the IOL eyelet and then passed behind the iris knuckle to emerge at the inner lip of the scleral tunnel (Fig. 1). After ensuring that there is no incarceration of iris tissue in the suture, the iris is reposited, and the leading haptic is placed on the capsular remnants. (The placement of the leading haptic can also be done prior to passing the fixation suture.) The IOL is then re-grasped with Kelman McPherson forceps and the trailing haptic to which the suture is tied, is released behind the iris so as to lie adjacent to the preplaced suture. The 9-0 polypropylene suture is pulled so as to gently center

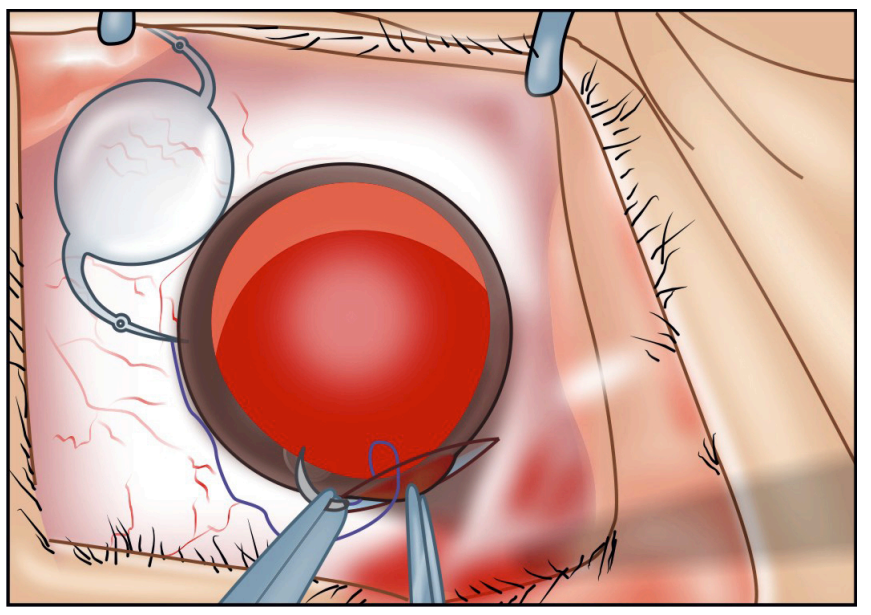

Fig. (1). The tip of the curved needle of 9-0 polypropylene to which the IOL eyelet is fastened is then passed behind the iris knuckle to emerge at the inner lip of the scleral tunnel. 
the IOL since too much pull can shift the IOL towards the wound. The needle with the 9-0 polypropylene is passed through the inner scleral lip and a loop is created to tie the suture on to itself (Figs. 2, 3). The anterior chamber is washed the wound is closed with one 10-0 nylon suture and the side port is hydrated. The conjunctiva is repositioned.

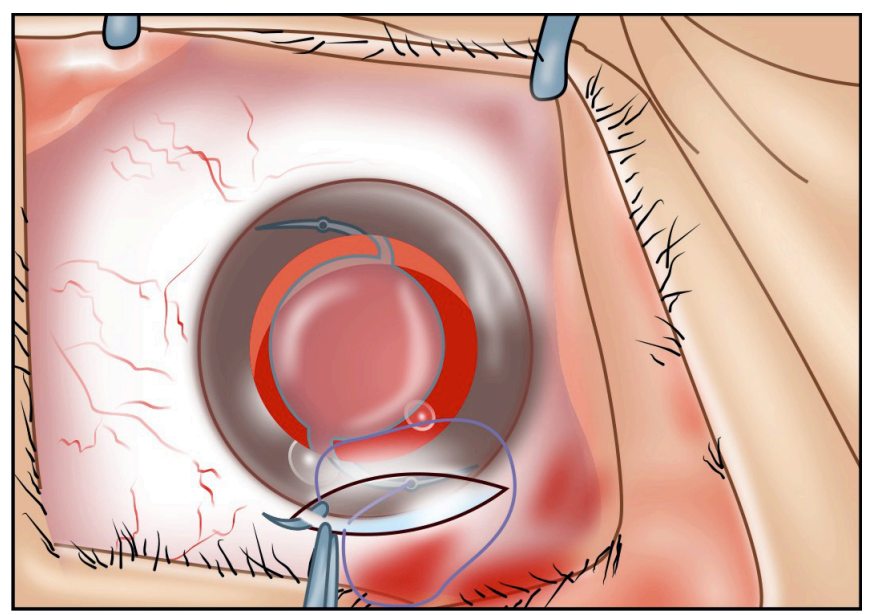

Fig. (2). The leading haptic is placed on the remaining capsule and the needle on 9-0 polypropylene (see above) is passed through the inner scleral lip.

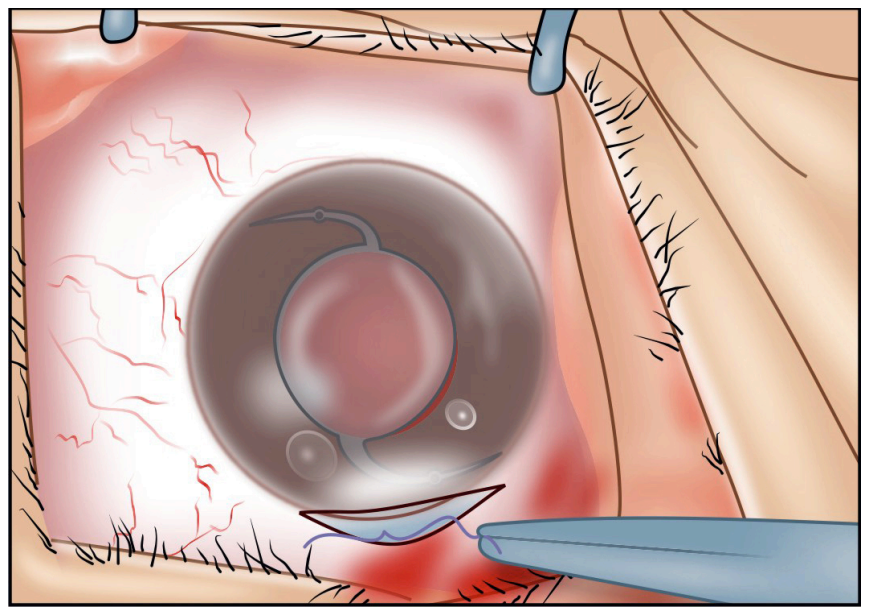

Fig. (3). The 9-0 polypropylene suture is tied on to itself.

The postoperative treatment is the same as for routine cataract surgery.

\section{RESULTS}

In a retrospective analysis of 10 patients who underwent single loop fixation using this technique performed by a single surgeon (RG), the average IOL tilt as measured by Visante ${ }^{\mathrm{TM}}$ OCT, Zeiss (software version 3.0.1.8) was $0.94^{\circ}$ at $180^{\circ}$ and $0.49^{\circ}$ at $90^{\circ}$ (Fig. 4).

\section{DISCUSSION}

Scleral fixation is performed using two or four point fixation to ensure IOL center position [4]. Multiple passes however increase surgical duration, predispose to infection and to the possibility of injuring the tissues during needle passage.
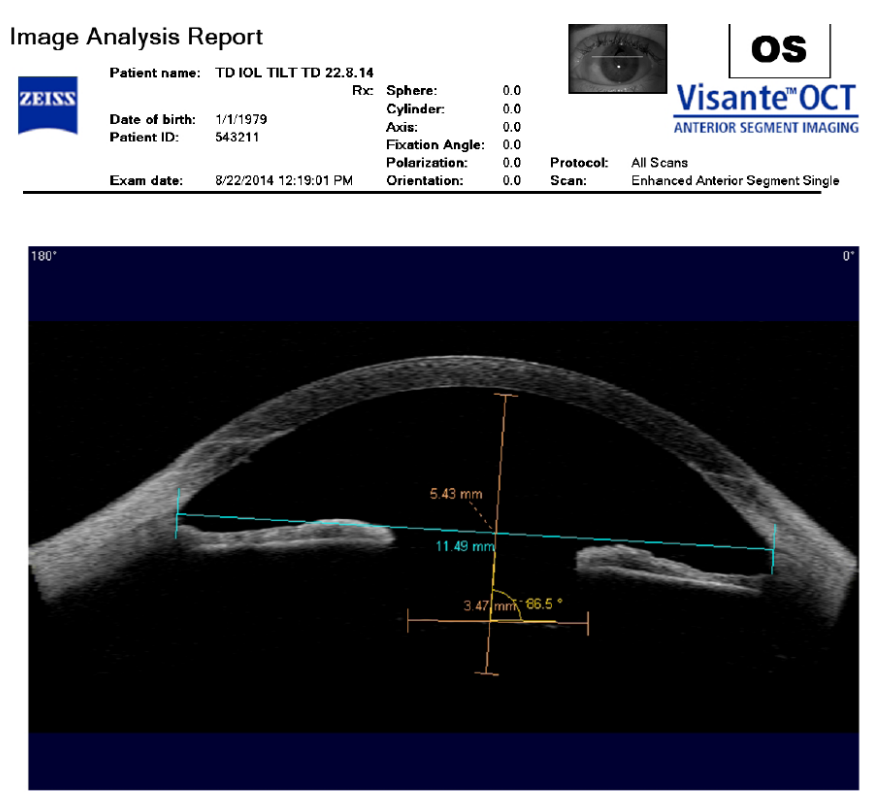

Fig. (4). The IOL tilt measured at $180^{\circ}$ by Visante ${ }^{\mathrm{TM}}$ OCT, Zeiss.

Suture erosion through the half thickness scleral flaps is a common complication [5]. Innovation such as tucking in of haptics in the scleral tunnel followed by sealing with fibrin glue [6] is associated with the risk of transmitted disease from pooled and single-donor blood donors [7]. In our technique, no extra port is created for fixing the IOL; also the knot is buried within the wound, so there is no chance of suture erosion.

In our study, lens centering is achieved by fixing the trailing loop $180^{\circ}$ away from the site of capsular remnants, the latter serving as a scaffold for placing the leading haptic. In contrast Yarangumeli et al. in their series of single haptic fixation in 6 patients, had one IOL tilt because they fixed all IOLs at 12 o clock irrespective of the site of capsular remnants [3].

Our procedure should not be performed in patients with less than $50 \%$ peripheral capsular rim as the stability of the IOL is jeopardized. The surgery can be performed at the same sitting in cases with posterior capsular tear, but becomes easier if done after a month of primary surgery as the capsule becomes fibrosed and provides a better scaffold.

In patients with absent capsular support between 5 and 7 o'clock position, the IOL has a tendency to shift due to the effect of gravity and are not suitable for single point fixation. Too tight a pull on the suture holding the haptic results in IOL getting off-center and tilted. So the suture should be just taut enough so as to keep the IOL centered. In our series none of the patients had more than a $2^{\circ}$ tilt.

The authors prefer to use a scleral fixated lens (PMMA) for this technique; however a closed loop foldable IOL can also be used with the suture being secured at the highest point on the haptic and not at the tip. In patients with intact capsule but generalized zonular laxity, a single loop fixation can be used as a safeguard for subsequent IOL drop.

To conclude, our technique of a single loop fixation can be safely practiced in the presence of at least $50 \%$ peripheral capsular rim, with at least some part present inferiorly. 


\section{CONFLICT OF INTEREST}

The authors confirm that this article content has no conflict of interest.

\section{ACKNOWLEDGEMENTS}

Declared none.

\section{REFERENCES}

[1] Holland EJ, Daya SM, Evangelista A, et al. Penetrating keratoplasty and transscleral fixation of posterior chamber lens. Am J Ophthalmol 1992; 114: 182-7.
[2] Por YM, Lavin MJ. Techniques of intraocular lens suspension in the absence of capsular/zonular support. Surv Ophthalmol 2005; 50(5): 429-62.

[3] Yarangumeli A, Alp MN, Kural G. Single-suture scleral fixation of subluxated foldable intraocular lenses. Eur J Ophthalmol 2012; 22(4): 547-53.

[4] Moawad AI, Ghanem AA. One-haptic fixation of posterior chamber intraocular lenses without scleral flaps. J Ophthalmol 2012; 2012: 891839.

[5] Solomon K, Gussler JR, Gussler C, Van Meter WS. Incidence and management of complications of transsclerally sutured posterior chamber lenses. J Cataract Refract Surg 1993; 19(4): 488-93.

[6] Sinha R, Bali SJ, Sharma N, Titiyal JS. Fibrin glue-assisted fixation of decentered posterior chamber intraocular lens. Eye Contact Lens 2012; 38(1): 68-71.

[7] Panda A, Kumar S, Kumar A, Bansal R, Bhartiya S. Fibrin glue in ophthalmology. Ind J Ophthalmol 2009; 57(5): 371-9.

(C) Malik et al.; Licensee Bentham Open.

This is an open access article licensed under the terms of the Creative Commons Attribution-Non-Commercial 4.0 International Public License (CC BY-NC 4.0) (https://creativecommons.org/licenses/by-nc/4.0/legalcode), which permits unrestricted, non-commercial use, distribution and reproduction in any medium, provided the work is properly cited. 